\title{
STRUCTURE OF UNDEREXPANDED ROUND AIR JETS SUBMERGED IN WATER
}

\author{
E. Loth and G. M. Faeth \\ Department of Aerospace Engineering, University of Michigan, Ann Arbor, MI 48109-2140, U.S.A.
}

(Received 29 January 1988; in revised form 27 December 1988)

\begin{abstract}
The structure and mixing properties of round turbulent air jets submerged in still water were studied experimentally, considering subsonic and sonic jet exit conditions with underexpansion ratios as high as $8: 1$. Measurements included the following mean properties: static pressure distributions along the axis, void-fraction distributions and entrainment rates. Confirming earlier findings, static pressure measurements showed the presence of a shock-wave-containing external-expansion region for underexpanded air jets in water, similar to the external-expansion region of underexpanded air jets in air. Increasing underexpansion ratios tended to stabilize large-scale unsteadiness observed for subsonic conditions, however, enhanced mixing near the jet exit, attributed to unsteadiness, persisted to the highest underexpansion ratios tested. The fully-developed portion of the flow exhibited widths based on void fractions that were roughly twice as large as widths based on scalar properties in single-phase jets; this behavior is caused by the strong sensitivity of void fractions to mixing levels, due to the large density ratio of the flow. Measurements were compared with predictions based on the locally-homogeneous-flow approximation of multiphase flow and the equivalent-adapted jet approximation for underexpanded jets. Predictions were encouraging but were sensitive to the specification of initial conditions and effects of enhanced mixing due to unsteadiness close to the jet exit.
\end{abstract}

Key Words: gas/liquid jets, compressible jets

\section{INTRODUCTION}

This investigation considered the structure and mixing properties of vertical round noncondensing gas jets in liquids, motivated by applications for metal processing, direct-contact condensors, gas dissolution systems, reservoir destratification systems, liquid-metal combustors and nuclear reactor pressure-suppression systems-among others. The main objective was to provide new measurements of flow structure. However, the measurements were also used to evaluate past proposals for analyzing the process which were based on the locally-homogeneous-flow (LHF) approximation of multiphase flow.

Gas jets submerged in liquids are complicated by effects of unsteadiness at low flow rates, similar to the pulsitile behavior of highly-buoyant single-phase flows. This involves oscillatory release of gas which can cause liquid to slug into the jet passage. The unsteadiness is accompanied by appreciable fluctuations of static pressure in the flow passage and beyond the jet boundaries, often resulting in excessive noise and vibration as well as blockage of the passage in cases where the gas reacts with the liquid (Kerney et al. 1972; Chan 1974; Avery \& Faeth 1974).

Effects of unsteadiness can be reduced by increasing gas flow rates in the passage, frequently leading to operation with underexpanded jets, where the flow is sonic and the static pressure is greater than the ambient pressure at the jet exit. It is well-known that static pressure equalization for underexpanded gas jets in gases occurs in an external-expansion region near the jet exit-involving shock waves and other compressible-flow phenomena (Shapiro 1954). Moiseev (1962) and Surin et al. (1983) observed that dynamic pressures along the axis of underexpanded gas jets in gases and liquids were similar. These observations suggest that a multiphase external-expansion region involving gas dynamic phenomena is present for underexpanded gas jets in liquids as well.

In view of the complexities of unsteadiness at low flow rates, and the presence of the external-expansion region at high flow rates, current understanding of gas injection into liquids is not very complete; nevertheless, the flow has received some attention in the past. Earlier work in this laboratory includes: noncondensing gas jets in liquids (Tross 1974); condensing gas jets in liquids (Kerney et al. 1972; Weimer et al. 1973; Chen \& Faeth 1982); and reacting gas jets in liquids (Avery \& Faeth 1974; Chen \& Faeth 1983). The earliest experimental studies were confined to gross 
parameters like the length of the vapor or gas-containing region of condensing or reacting jets (Kerney et al. 1972; Weimer et al. 1973; Avery \& Faeth 1974). Analysis was also undertaken based on an integral model of turbulence and the LHF approximation of multiphase flow theory (Soo 1967). The LHF approximation implies infinitely-fast interphase transport rates and local thermodynamic equilibrium between the phases, i.e. velocity differences between the phases are neglected and phase and chemical equilibrium are assumed to be maintained at each point within the flow. Gas-dynamic processes in multiphase flows are not well-understood; therefore, the complexities of the external-expansion region were avoided by defining effective-adapted-jet exit conditions, where the static pressure at the passage exit was the same as the ambient pressure. This approach was reasonably successful for correlating the length of the vapor- or gas-containing region of condensing jets (Kerney et al. 1972; Weimer et al. 1973; Avery \& Faeth 1974).

Tross (1974) measured mean void fractions, using an electrical conductivity probe, and dynamic pressures, using an impact probe, in adapted and underexpanded air jets in still water. The flow analysis was similar to Weimer et al. (1973) and Avery \& Faeth (1974). Unfortunately, the analysis was not very effective for predicting flow structure in spite of earlier success. This was attributed to the uncertainties of probe measurements in multiphase flows as well as problems of treating a rapidly-developing flow, having a large density ratio, with an integral model (Tross 1974).

Subsequent work in this laboratory considered a higher-order turbulence model in an effort to improve predictions (Chen \& Faeth 1982, 1983). The conserved-scalar formalism of Bilger (1976) and Lockwood \& Naguib (1975) was used, which had provided a successful treatment of constant density, variable density and combusting turbulent jets during other work in this laboratory (Jeng \& Faeth 1984). The LHF approximation was adopted, while only flow rates slightly in excess of the sonic condition were considered to avoid problems of external expansion. A key feature of the combined use of the LHF and the conserved-scalar approximations is that scalar properties (void fraction, density etc) are only functions of the mixture fraction (the fraction of mass at a point which originated from the injector). These functions, called state relationships, can be found from straightforward adiabatic mixing or adiabatic reaction calculations, typical of fundamental thermodynamic theory. Use of the turbulence model and LHF approximations was successful for predicting the length of the vapor- or gas-containing region of both condensing and reacting jets, with the empirical parameters of the turbulence model fixed at values appropriate for constantdensity single-phase jets (Chen \& Faeth 1982, 1983). The same approach has been evaluated for a variety of other multiphase jets; see Faeth (1987) and references cited therein. In general, the approach was reasonably successful in dense multiphase flows but tended to overestimate the rate of development of dilute dispersed flows where finite interphase transport rates become important and the LHF approximation fails.

Related studies by other paralleled activities in this laboratory. Early work by Cumo et al. (1978), Kudo et al. (1974), Lee et al. (1979) and Young et al. (1974) involved measurement of only the gross features of condensing jets, like the length of the vapor-containing region. Relevant portions of this data were used to evaluate LHF analysis of condensing jets (Chen \& Faeth 1982). Bakaklevskii \& Chekhovich (1978) report temperature and dynamic pressure profiles in condensing plane jets in coflow, however, measurement accuracy and flow conditions are too uncertain to provide more than a rough guide to structure. Chan (1974), Chun \& Sonin (1984), Lambier \& Chow (1984) and Simpson \& Chan (1982) measured static pressures near the passage exit for subsonic condensing jets, finding the large pressure pulsations, indicative of unsteady flow, that were mentioned earlier. Structure measurements are more numerous for noncondensing jets but most results are limited to diluted bubbly flows far from the passage exit (Abdel-Aal et al. 1966; Mahalingen et al. 1976; Ohba et al. 1977; Ohba 1979). Past studies of processes near the passage exit generally involve the use of probes or other intrusive techniques, for example: the dynamic pressure measurements of Moiseev (1962) and Surin et al. (1983); measurements of void diameter using a passage plate by Bell et al. (1972); and measurments of entrainment using the water overflow technique by Bell et al. (1972) and Carreau et al. (1985).

The main objective of the present investigation was to provide new nonintrusive measurements of the structure of adapted and underexpanded air jets in still water. In addition to flow visualization, this included measurements of mean void fractions, using $\gamma$-ray absorption; and entrainment velocities (rates) using laser-Doppler anemometry. Static pressures were also mea- 
sured, using probes, to examine the gas-dynamic properties of the external-expansion region as well as acoustic phenomena associated with unsteadiness near the passage exit. The new structure measurements, supplemented by the earlier measurements of Tross (1974), were used to evaluate predictions based on past proposals for analyzing this flow, e.g. use of the LHF and effectiveadapted-jet approximations (Chen \& Faeth 1982; Faeth 1987).

The paper begins with a description of experimental and theoretical methods. Results are then considered, treating flow definition, structure, entrainment and the comparison between predictions and measurements. The discussion which follows is brief, more details and a complete tabulation of data can be found in Loth (1988).

\section{EXPERIMENTAL METHODS}

\subsection{Apparatus}

Measurements were carried out in a water-filled tank $(1 \times 2 \times 2 \mathrm{~m})$ high which had glass side walls and was open at the top. The injector was directed vertically upward and could be transversed in three directions with vertical and horizontal positioning accuracies of 1 and $0.1 \mathrm{~mm}$. The injector was located near the center of the tank and was generally at least $650 \mathrm{~mm}$ below the liquid surface while the liquid was $1.5 \mathrm{~m}$ deep. Flow disturbances at the liquid surface were controlled with wave dampers, yielding wave heights $<75 \mathrm{~mm}$ and pressure disturbances near the jet exit $<2 \mathrm{kPa}$.

The injector consisted of a plenum, a honeycomb flow straightener ( $1.6 \mathrm{~mm}$ cells, $25 \mathrm{~mm}$ long) and a calming section, all having a diameter of $32 \mathrm{~mm}$, followed by a constant-radius converging section to a constant-diameter flow passage. Two jet passages were used having inside diameters of 4.9 and $11.0 \mathrm{~mm}$ and lengths of $230 \mathrm{~mm}$ to yield nearly fully-developed pipe flow at the exit. The passages extended into the water as tubes for most of their length and were tapered from the outside to a sharp edge at the exit. Static pressures were measured in the plenum and one diameter upstream of the exit.

The air supply was drawn from laboratory facilities which were filtered and dried to a dewpoint $<240 \mathrm{~K}$. Air flow rates were controlled with pressure regulators and metered with critical-flow nozzles having diameters of 2.43 and $7.62 \mathrm{~mm}$ (Flow Engineering Co., $0.1 \%$ accuracy). Pressures on the upstream side of the critical-flow nozzles were measured with a $250 \mathrm{~mm}$ dia Heisse pressure gage (0-2 $\mathrm{MPa}$ range).

\subsection{Instrumentation}

\section{Static pressure}

Static pressures were measured along the axis of the jets using an arrangement similar to early studies of underexpanded gas jets in gases, see Eggers (1966) and references cited therein. These measurements were carried out with the $11.0 \mathrm{~mm}$ dia passage. The static pressure probe consisted of a $1 \mathrm{~mm}$ dia stainless-steel tube with the upstream end closed and a $0.4 \mathrm{~mm}$ dia static pressure tap. The static pressure tap was located so that it could be traversed over the region $-1 \leqslant x / d \leqslant 7$, with a positioning accuracy of $1 \mathrm{~mm}$, where $x$ is the distance from the jet exit and $d$ is the passage exit diameter. The probe was centered along the axis with two supports: one within the passage at $x / d=-6$, the other in the bath at $x / d=13$. A small air purge was used to keep the proble free of water when measuring air jets in water. Static pressures were read with a $250 \mathrm{~mm}$ dia Heisse pressure gage (0-2 $\mathrm{MPa})$ and are estimated to have experimental uncertainties $(95 \%$ confidence) $<30 \%$ of the pressure difference between the jet and the water bath (Loth 1988).

It is well-known that acoustic feedback can cause instabilities which enhance mixing of underexpanded gas jets in gases (Sherman et al. 1976). This effect was studied using a Spartan model 110-8459 hydrophone ( $2 \mathrm{~dB}$ response in the range $1-40 \mathrm{kHz})$ during tests with the $4.9 \mathrm{~mm}$ dia passage. The hydrophone was positioned in the bath at $x / d=6$ and $r / d=6$, where $r$ is the distance from the jet axis. The hydrophone output was processed by averaging 100 power spectral density distributions obtained with a rectangular window and a resolution of $40 \mathrm{~Hz}$. 


\section{Void fractions}

Distributions of time-averaged void fractions were measured using $\gamma$-ray absorption. Absorption measurements for chord-like paths through the flow, at a given streamwise distance, were deconvoluted to find radial distributions of the time-averaged void fraction. $\gamma$-rays were obtained from a ${ }^{57} \mathrm{Co}$ source (Amersham Inc., $2 \mathrm{mCi}$, 271-day half-life) placed in a $\mathrm{Pb}$ casket, with an output beam of $\gamma$-rays directed across the flow using a $\mathrm{Pb}$ collimator ( $2 \mathrm{~mm}$ dia; $13 \mathrm{~mm}$ long). After traversing the flow, the beam passed through a $\mathrm{Pb}$ aperture $(2,3$ and $5 \mathrm{~mm}$ dia, depending on the jet width; $13 \mathrm{~mm}$ long) to the detector (EG \& G model 905-1 NaI scintillator and photomultiplier). The detector signal was preamplified and then processed by an EG \& G model 590 A single-channel analyzer and amplifier, and an EG \& G model 974 timer/counter. The output counts of the timer/counter were then collected and stored, for various sampling times, using an IBM-AT computer. The absorption window of the detector was $114-128 \mathrm{keV}$, to capture the $122 \mathrm{keV}$ emission of the source while eliminating low-energy background radiation. The source and detector were located on either side of the jet flow, submerged in the tank in waterproof housings.

At each axial position, absorption measurements were obtained for $20-40$ horizontal chord-like paths through the flow. Roughly 10,000 counts were accumulated for each path which required sampling times of 8-50 min. The linear absorption coefficient of $\gamma$-rays for air is about four orders of magnitude smaller than for water; therefore, the logarithm of the counting rate is proportional to the fraction of air in the radiation path. Assuming axisymmetric flow, the absorption measurements were deconvoluted, following Santoro et al. (1981), to obtain radial profiles of the time-averaged void fraction.

Bias errors of the void-fraction measurements are influenced by whether liquid laminae in the absorption path are parallel to normal to the path (Ohba 1979). Low-intensity ratios reduced uncertainties due to this effect to $<5 \%$, based on the findings of Ohba (1979). Dynamic bias errors, due to turbulent fluctuations of void fractions along the path, were estimated by deconvolutions through a linearized error analysis, which showed bias errors of $<5 \%$.

The main source of experimental uncertainty was amplification of count rate uncertainty during deconvolution. This was calculated using the matrix model of Limbaugh \& Kneile (1984) for results obtained by deconvolution of data with a prescibed uncertainty. This resulted in experimental uncertainties ( $95 \%$ confidence) at the centerline of $<20 \%$, with relative uncertainties increasing inversely proportional to the indicated mean void function at off-axis positions. Assessment of errors, based on retrieving a mean void fraction of unity for several positions in the jet air core, confirmed uncertainty estimates with these limits (Loth 1988).

\section{Velocities}

A conventional dual-beam, frequency-shifted, forward-scatter, laser-Doppler anemometer (LDA) was used to measure mean and fluctuating streamwise velocities at the injector exit (with the tank empty) and mean jet entrainment velocities in the liquid. The uncertainties of the entrainment velocity measurements $(95 \%$ confidence) are estimated to be $<10 \%$ (Loth 1988).

\section{Entrainment}

Jet entrainment rates, defined as the rate of increase of the mass flow rate of the jet with streamwise distance, were obtained from the entrainment velocity measurements. These measurements were made along a jet boundary outside the gas-containing region at a radial distance $r_{\infty}$ while correcting for low levels of streamwise velocity, $u_{\infty}$, in the region where accurate measurements of radial velocity, $v_{\infty}$, could be made. Based on these measurements, the rate of increase of the mass flow rate of the jet, $\dot{m}$, with streamwise distance is

$$
\frac{\mathrm{d} \dot{m}}{\mathrm{~d} x}=2 \pi r_{\infty} \rho_{\infty}\left(\bar{u}_{\infty} \tan \beta_{\infty}-\bar{v}_{\infty}\right)
$$


Table 1. Summary of the test conditions ${ }^{\mathrm{a}}$

\begin{tabular}{|c|c|c|c|c|c|}
\hline$\dot{m} / \dot{m}_{\mathrm{s}}$ & $\rho_{\mathrm{e}}(\mathrm{kg} / \mathrm{m})^{3}$ & $\dot{m}_{\mathrm{e}}(\mathrm{g} / \mathrm{s})$ & $\dot{M}_{\mathrm{e}}(\mathrm{N})$ & $\operatorname{Re} / 10^{5 b}$ & $\mathrm{Ri} / 10^{-4 \mathrm{c}}$ \\
\hline \multicolumn{6}{|c|}{$4.9 \mathrm{~mm}$ injector } \\
\hline 0.6 & 1.36 & 5.28 & 1.11 & 0.80 & 8.14 \\
\hline 1.0 & 1.52 & 8.68 & 2.73 & 1.34 & 3.31 \\
\hline 2.0 & 3.06 & 17.5 & 7.42 & 3.62 & 1.21 \\
\hline 4.0 & 6.12 & 35.8 & 16.7 & 8.01 & 0.54 \\
\hline 8.0 & 12.3 & 71.7 & 35.9 & 17.2 & 0.25 \\
\hline \multicolumn{6}{|c|}{$11.0 \mathrm{~mm}$ injector } \\
\hline 0.6 & 1.36 & 26.3 & 5.53 & 1.80 & 18.5 \\
\hline 1.0 & 1.52 & 43.8 & 13.8 & 3.01 & 7.41 \\
\hline 2.0 & 3.06 & 87.7 & 37.3 & 8.16 & 2.73 \\
\hline 4.0 & 6.12 & 174.0 & 83.6 & 18.5 & 1.22 \\
\hline
\end{tabular}

${ }^{a}$ Initial conditions for an air jet injected vertically upward in still water having a temperature of $294 \pm 2 \mathrm{~K}$ an ambient pressure at the jet exit of $107.5 \pm 0.7 \mathrm{kPa}$ and an air stagnation temperature of $295 \pm 2 \mathrm{~K}$.

${ }^{\mathrm{b}} \operatorname{Re}=\rho_{\mathrm{e}} \dot{M}_{\mathrm{e}} d /\left(\mu_{\mathrm{e}} \dot{m}_{\mathrm{e}}\right)$.

${ }^{\mathrm{c}} \mathrm{Ri}=\left(\rho_{x} / \rho_{\mathrm{e}}-1\right) \operatorname{ad}\left(\dot{m}_{\mathrm{e}} / \dot{M}_{\mathrm{e}}\right)^{2}$.

where $\rho$ denotes density, $\beta_{\infty}$ is the angle between the jet boundary and the axis, and an overbar denotes a time average. This yields the following expression for the dimensionless entrainment coefficient:

$$
\frac{\frac{\mathrm{d} \dot{m}}{\mathrm{~d} x}}{\left(\dot{m}_{\mathrm{e}} u_{\mathrm{e}} \rho_{\infty}\right)^{1 / 2}}=\frac{2 \pi r_{\infty} \rho_{\infty}^{1 / 2}\left(\bar{u}_{\infty} \tan \beta_{\infty}-\bar{v}_{\infty}\right)}{\dot{M}_{\mathrm{e}}^{1 / 2}},
$$

where properties at the injector exit are denoted with a subscript e and $\dot{M}_{\mathrm{e}}$ is the streamwise thrust on the passage.

The term involving $\bar{u}_{\infty}$ in [1] and [2] contributed $<10 \%$ to the entrainment while entrainment rates were relatively independent of the location of $r_{\infty}$ outside the gas-containing region. Estimated experimental uncertainties ( $95 \%$ confidence) of the entrainment measurements were $<20 \%$, largely governed by uncertainties of the velocity measurements and effects of locating $r_{\infty}$ (Loth 1988).

\subsection{Test Conditions}

The test conditions are summarized in table 1 , where $\dot{m} / \dot{m}_{\mathrm{s}}$ is the underexpansion ratio (the ratio of the mass flow rate to the adapted sonic mass flow rate at the passage exit), $a$ is the acceleration of gravity and $\mu_{\mathrm{e}}$ is the viscosity of air at the passage exit. Formulas defining the jet Reynolds number, $\mathrm{Re}$, and the jet Richardson number, $\mathrm{Ri}$, also appear in the table. Flow rates varied from subsonic jets to highly underexpanded sonic jets $\left(\dot{m} / \dot{m}_{\mathrm{s}}\right.$, in the range 0.6-8.0). For $\dot{m} / \dot{m}_{\mathrm{s}} \geqslant 1$, the ratios of the passage exit to ambient static pressures are equal to $\dot{m} / \dot{m}_{\mathrm{s}}$. Flow Reynolds numbers are quite high, ca. $10^{5}$, with approximately fully-developed pipe flow at the passage exit; therefore, the jets were turbulent. Richardson numbers were relatively low, ca. $10^{-4}$; therefore, effects of buoyancy were small in the external-expansion region near the passage exit. However, mixing is rapid for air injection into water and the large density difference between water and air causes significant effects of buoyancy farther downstream, $x / d>20$.

\section{THEORETICAL METHODS}

\subsection{General Description}

The analysis was similar to the approach proposed by Chen \& Faeth $(1982,1983)$ to treat flows of this type. The method has been evaluated in this laboratory using structure measurements for a wide range of multiphase jets [see Faeth (1987) and references cited therein]: the objective of present work was to extend this evaluation to underexpanded gas jets in liquids. The formulation appears in Faeth (1987) and will only be described briefly here.

The three major assumptions of the analysis are as follows: (1) use of the LHF approximation to treat multiphase flow effects; (2) use of effective-adapted-jet conditions to treat the external-expansion region of underexpanded jets; and (3) use of a higher-order turbulence model to treat 
turbulent mixing. The LHF approximation implies negligible relative velocities between the phases and local thermodynamic equilibrium; therefore, the flow is treated like a single-phase fluid having large density variations due to changes in gas concentrations while separated-flow parameters, like drop and bubble size distributions, do not enter the formulation. Consistent with present limited knowledge concerning the structure of gas jets in liquids, it seems prudent to evaluate the performance of LHF analysis, as a baseline, before undertaking the additional complications of separated-flow analysis. Furthermore, recent evaluations of the LHF approximation suggest reasonably good performance in the near-injector region of sprays and bubbly jets (Faeth 1987; Ruff et al. 1989, Sun \& Faeth 1986): this provides additional motivation for examination of the approach here.

The effective-adapted-jet approximation is frequently used to avoid the complexities of treating gas-dynamic phenomena in external-expansion regions when estimating turbulent mixing for both single- and multiphase flows (Avery \& Faeth 1974; Birch et al. 1984, 1987; Chuech et al. 1989; Kerney et al. 1972; Weimer et al. 1973). Recent evaluations have also shown that the approach is reasonably effective for estimating the structure of underexpanded gas jets in gases, even close to the external-expansion region (Birch et al. 1984, 1987; Chuech et al. 1989). Since present measurements, and those of Surin et al. (1983), suggest that external-expansion regions of underexpanded gas jets in liquids are smaller than for underexpanded gas jets in gases, the region where the approximation is useful should be even larger for the present flows.

Due to the high Reynolds numbers of the present flows, some degree of modeling must be accepted to treat their mixing properties. Consistent with past work on gas jets in liquids and related multiphase jets in this laboratory (Chen \& Faeth 1982, 1983; Faeth 1987; Ruff et al. 1989; Sun \& Faeth 1986), turbulent mixing was treated using a simplified $k-\epsilon-g$ turbulence model. The approach is similar to an early recommendation by Lockwood \& Naguib (1975) but is extended to use mass-weighted (Favre) averages to simplify treatment of density fluctuations as recommended by Bilger (1976). Empirical constants were based on calibrations for constant- and variable-density single-phase round jets using the Favre-averaged formulation (Jeng \& Faeth 1984), however, the values are not very different from Lockwood \& Naguib (1975). The approach is plausible since the present flows are geometrically simple high Reynolds number turbulent flows for which turbulence models were developed and have been reasonably successful. Furthermore, recent evaluation of this approach for the near-injector region of large-scale pressure-atomized sprays has been reasonably successful (Ruff et al. 1989), suggesting some capability to treat large density ratios, comparable to the present flows, as well.

Other major assumptions of the analysis are as follows: steady (in the mean) axisymmetric turbulent jet with no swirl; boundary-layer approximations apply; equal exchange coefficients of all species, phases and heat; buoyancy considered in the governing equations for all mean quantities but ignored in the governing equations for turbulence quantities; and effects of mean kinetic energy, viscous dissipation and compressibility are ignored. The first assumption is a condition of the experiments while use of the boundary-layer approximations is not an issue for nonswirling jets. Assuming equal exchange coefficients of all species, dispersed phases and heat is widely accepted for high Reynolds number turbulent flows where turbulent transport dominates the flow properties (Lockwood \& Naguib 1975; Bilger 1976; Jeng \& Faeth 1984; Faeth 1987). Neglecting buoyancy in the governing equations for turbulence quantities minimizes empiricism while past work shows that the effect of this approximation is small with respect to mean properties (Jeng \& Faeth 1984). Finally, underexpanded jets involve supersonic flow downstream of the passage exit so that neglecting effects of mean kinetic energy, viscous dissipation and compressibility is clearly questionable. However, the external expansion region is not large and is only treated approximately using the effective-adapted-jet approach; therefore, the added complications of treating these effects are not justified.

\subsection{Formulation}

Under present assumptions, all scalar properties may be represented solely as functions of mixture fraction, called state relationships, and the conserved-scalar formalism can be used (Bilger 1976). This involves solving governing equations for conservation of mass, mean momentum, mean mixture fraction, turbulence kinetic energy, the rate of dissipation of turbulence kinetic 
energy and mixture fraction fluctuations squared. The details of the formulation (including all empirical constants, boundary conditions, and the computation of Favre- and time-averaged scalar properties) are described elsewhere (Faeth 1987; Jeng \& Faeth 1984; Sun \& Faeth 1986).

Several effective-adapted-jet approximations have been proposed to treat the mixing properties of underexpanded jets while avoiding the complications of the external-expansion region, see Chuech et al. (1989) for a review of methods proposed thus far. The divergent-nozzle approach of Kerney et al. (1972) was used during the present investigation. This involves replacing the actual external-expansion process by isentropic flow to the ambient pressure, and applying the new diameter, velocities etc. of the flow at the exit plane of the actual passage-ignoring the presence of any virtual origin. This approach has been effective for estimating the mixing properties for injection of gases into gases, and conserves mass, momentum and energy for the flow (Chuech $e t$ al. 1989). The character of the flow, fully-developed pipe flow, was preserved in this process as recommended by Chuech et al. (1989), however, use of either slug flow or fully-developed flow had a negligible effect on computed results in the region where measurements were made.

By definition, the mixture fraction is unity across the jet exit, yielding standard initial conditions. Present measurements, however, suggested enhanced mixing very close to the jet due to effects of intrinsic unsteadiness. To represent this effect, a partially-mixed initial condition was also considered to obtain a better fit of void-fraction distributions and entrainment rates near the passage exit. This involved a void fraction of unity across the core of the flow followed by a sinusoidal variation of void fraction to the ambient value of zero at the edge of the flow in the region $0.4 \leqslant r / d \leqslant 0.6$.

\subsection{Computations}

The governing equations were integrated using a modified version of the GENMIX algorithm due to Spalding (1977). The large density variations of the present multiphase flows required a finer numerical grid than is needed for single-phase flows. Results reported in the following used $\mathbf{3 6 0}$ cross-stream grid nodes, with streamwise step sizes chosen to be $<0.2 \%$ of the current flow width, or an entrainment increase of $<0.2 \%$ whichever was limiting. Doubling the number of nodes in the mesh resulted a $<1 \%$ variation of flow properties; therefore, numerical closure was adequate, particularly in view of the other uncertainties of the analysis.

\section{RESULTS AND DISCUSSION}

\subsection{Flow Definition}

\section{Initial conditions}

Flow properties at the passage exit $(x / d=0.2)$ were measured for air injection into still air for all expansion ratios $\left(\dot{m} / \dot{m}_{\mathrm{s}}\right)$. Mean and fluctuating flow properties closely agreed with the properties of full-developed pipe flow for $\dot{m} / \dot{m}_{\mathrm{s}} \leqslant 1$, measured by Nikuradse \& Laufer and cited by Hinze (1975) and Schlichting (1979). For $\dot{m} / \dot{m}_{\mathrm{s}}>1$, there was evidence of acceleration of the flow near its edge, due to external expansion, since the measuring plane was slightly downstream of the exit. However, the results suggested the fully-developed pipe flow was still maintained at the exit.

\section{Flow visualization}

Motion pictures of the flow (at roughly 1000 pictures/s) gave evidence of large-scale unsteadiness for $\dot{m} / \dot{m}_{\mathrm{s}}=0.6$ and 1.0, similar to the observations of others (Kerney et al. 1972; Weimer et al. 1973; Chan 1974; Chun \& Sonin 1984; Lambier \& Chow 1984; Simpson \& Chan 1982). This involved random fluctuations of gas release (ca. 10-20/s) producing mushroom-like gas structures near the exit and the sudden appearance (ca. $1 \mathrm{~ms}$ ) of gas below the passage exit. This behavior was similar to the pulsitile release of gas near the source of strongly-buoyant single-phase plumes. The frequency of appearance of large-scale disturbances decreased with increasing $\dot{m} / \dot{m}_{\mathbf{s}}$ for underexpanded jets. Only occasional disturbances (typically 2-3/s) were observed for $\dot{m} / \dot{m}_{\mathrm{s}}=8$, lasting only a few milliseconds. They appear to be similar to the "reverse shocks" mentioned by Surin et al. (1983) and are associated with the external-expansion region, possibly involving interactions between shock waves and other gas-dynamic phenomena and the motion of gas-liquid 
interfaces. Plenum pressure fluctuations due to these disturbances decreased monotonically with increasing injector mass flow rates, e.g. maximum plenum pressure fluctuations were 1.2 and $0.2 \%$ at $\dot{m} / \dot{m}_{\mathrm{s}}$ of values 0.6 and 8 , respectively. The use of screens at the passage exit, and various injector exit configurations, injector angles (ranging from horizontal to vertically upward), liquid depths, plenum sizes and wave-damping methods, had no observable influence on the character of the large-scale unsteady disturbances.

\section{Acoustic feedback}

Hydrophone measurements were undertaken to determine whether unsteadiness observed near the jet exit was caused by acoustic feedback which is known to enhance mixing in underexpanded gas jets in gases (Sherman et al. 1976). Power spectra for air jets in air only indicated a resonance at $23 \mathrm{kHz}$ for $\dot{m} / \dot{m}_{\mathrm{s}}=2.0$ and a broad peak near $20 \mathrm{kHz}$ for $\dot{m} / \dot{m}_{\mathrm{s}}=3.0$. These relatively small effects of acoustic feedback are consistent with the present use of a protruding injector which provided little surface area to reflect sound waves (Sherman et al. 1976). Furthermore, no resonances were observed at all for underexpanded air jets in water, probably due to different acoustic velocities and damping effects of multiphase mixtures, which modify conditions required for resonance. Thus, unsteadiness observed for the present underexpanded gas jets in liquids does not appear to be caused by conventional mechanisms of acoustic feedback.

\subsection{Flow Structure}

\section{Static pressures}

Static pressures along the axis, for $\dot{m} / \dot{m}_{\mathrm{s}} \geqslant 1.0$, are illustrated in figure 1 . Results are shown for injection into both air and water, from the same $11.0 \mathrm{~mm}$ dia passage. The results for injection into air are very similar to the findings of Chuech et al. (1989) for underexpanded fully-developed pipe flows in still air. Static pressures along the axis exhibit a decaying oscillatory behavior due to the presence of shock cells that eventually decay away as the mixing layers near the edge of the flow reach the axis. The static pressure variations along the axis for injection of air into water are similar to those observed for injection of air into air, at least for the first few shock cells. Notably, Surin et al. (1983) observed similar agreement of oscillatory dynamic pressure variations along the axis of underexpanded gas jets in gases and liquids. This provides strong evidence that a shock-wave-containing external-expansion region is present for underexpanded gas jets in liquids. The main difference between the static pressure records for underexpanded air jets in air and in water is that the external-expansion region decays more rapidly for injection into water. This behavior is expected since turbulent mixing is more rapid when a low-density material is injected into a high-density environment (Chen \& Faeth 1982, 1983; Faeth 1987).

\section{Void fractions}

Time-averaged void fractions along the axis $\bar{\epsilon}_{c}$, are illustrated in figure 2. Results shown include present measurements for $d=4.9 \mathrm{~mm}$, the probe measurements of Tross (1974), for $\dot{m} / \dot{m}_{\mathrm{s}}=1.0$ and 2.0, and predictions (denoted the EEC model) using both standard and mixed initial conditions. The measurements of Tross (1974) are consistently lower than the present measurements in the region where they can be compared. This behavior is expected since all biases of probes tend to reduce observed void fractions (Tross 1974). It is felt that the present nonintrusive $\gamma$-ray absorption measurements are more reliable. The measurements show that mixing rates along the axis are progressively reduced as the underexpansion ratio is increased. This slower rate of mixing is the price that must be paid to obtain more stable injector operation by increasing $\dot{m} / \dot{m}_{\mathrm{s}}$.

Predictions agree reasonably well with present measurements in figure 2 , for $\dot{m} / \dot{m}_{\mathrm{s}} \geqslant 2$ where the effects of unsteadiness are reduced. Use of mixed initial conditions provide somewhat better agreement with measurements than the standard initial conditions at lower values of $\dot{m} / \dot{m}_{\mathrm{s}}$, however, both methods still underestimate mixing rates at $\dot{m} / \dot{m}_{3}=0.6$. This deficiency cannot be due to the LHF approximation, which invariably causes mixing rates of multiphase flows to be overestimated (Faeth 1987) while the effective-adapted-jet approximation is not used at $\dot{m} / \dot{m}_{\mathrm{s}}=0.6$. Thus, increased large-scale unsteadiness observed at low $\dot{m} / \dot{m}_{\mathrm{s}}$ is probably responsible for the enhanced mixing exhibited by the measurements. 


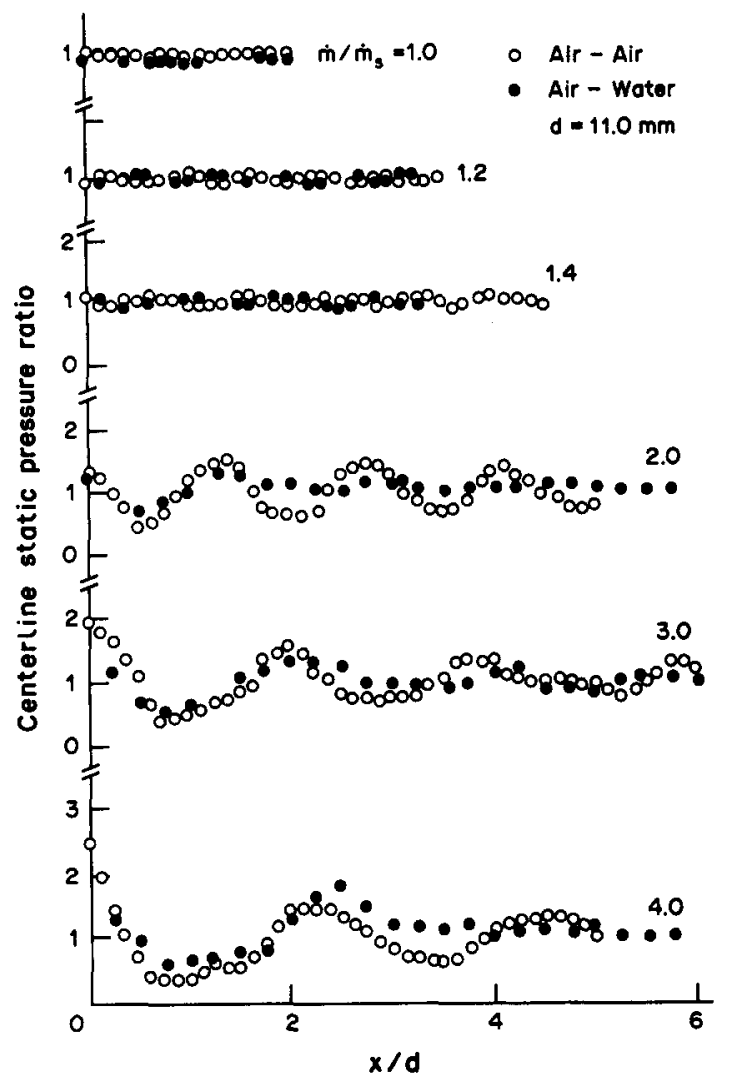

Figure 1. Mean static pressures along the axis for various mass flow ratios (11.0 $\mathrm{mm}$ dia passage).

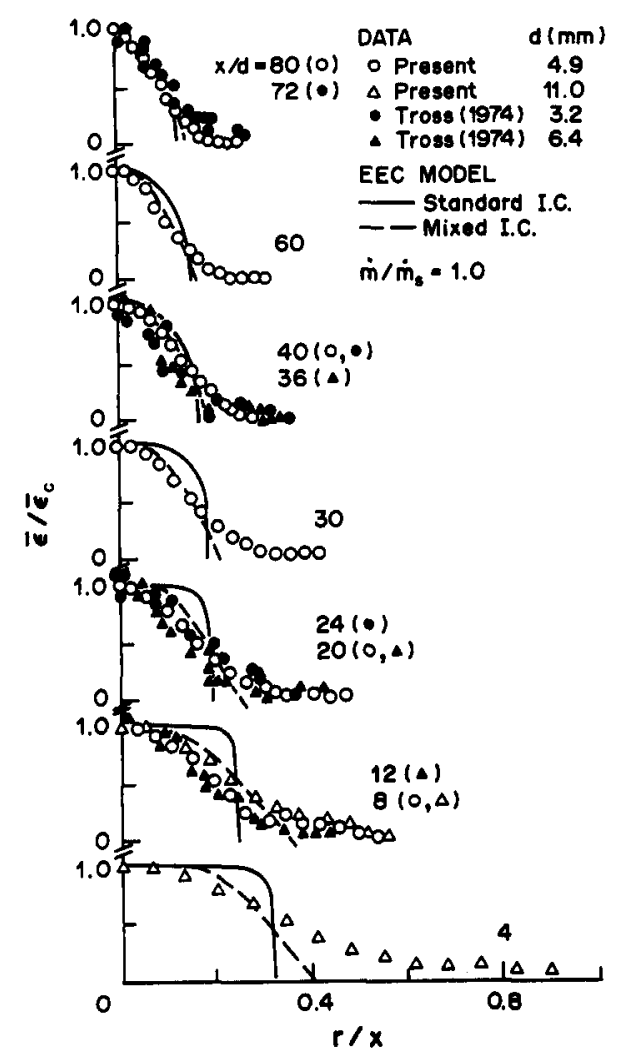

Figure 3. Time-averaged void fractions for a mass flow ratio of 1.0 .

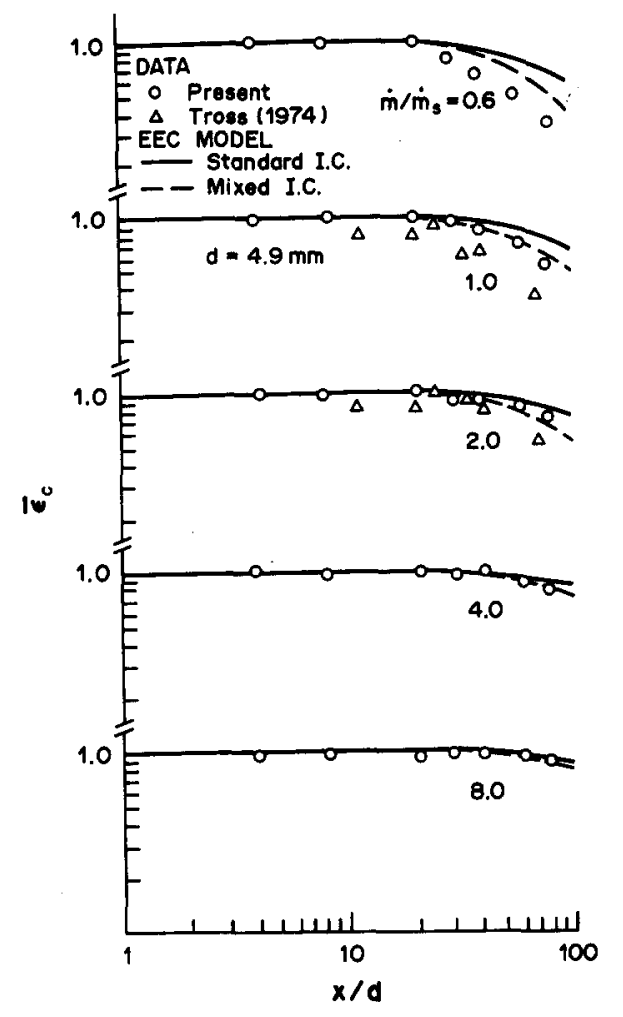

Figure 2. Time-averaged void fractions along the axis for various mass flow ratios.

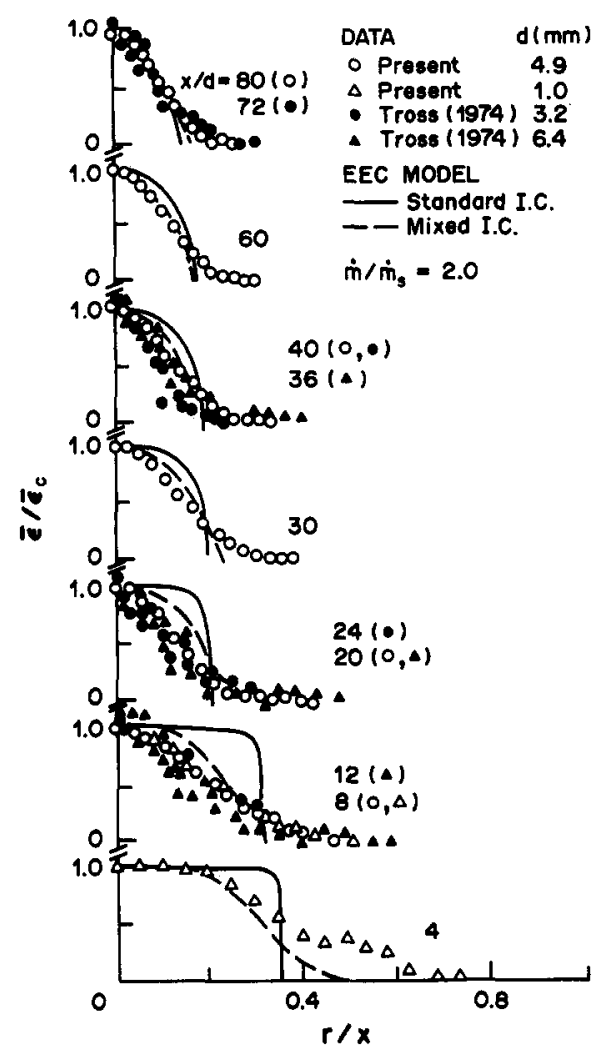

Figure 4. Time-averaged void fractions for a mass flow ratio of 2.0 . 


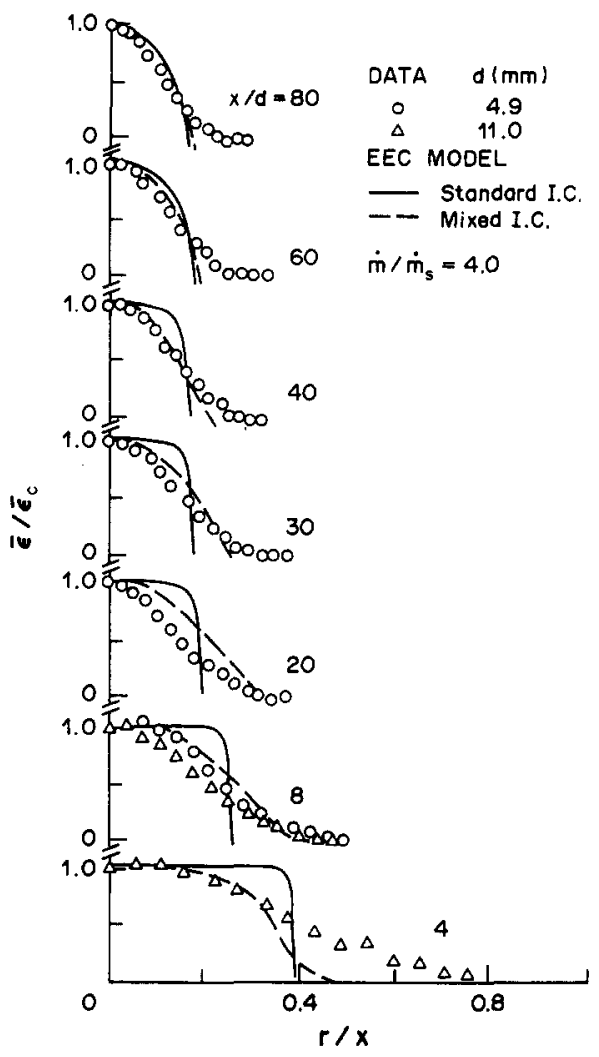

Figure 5. Time-averaged void fractions for a mass flow ratio of 4.0 .

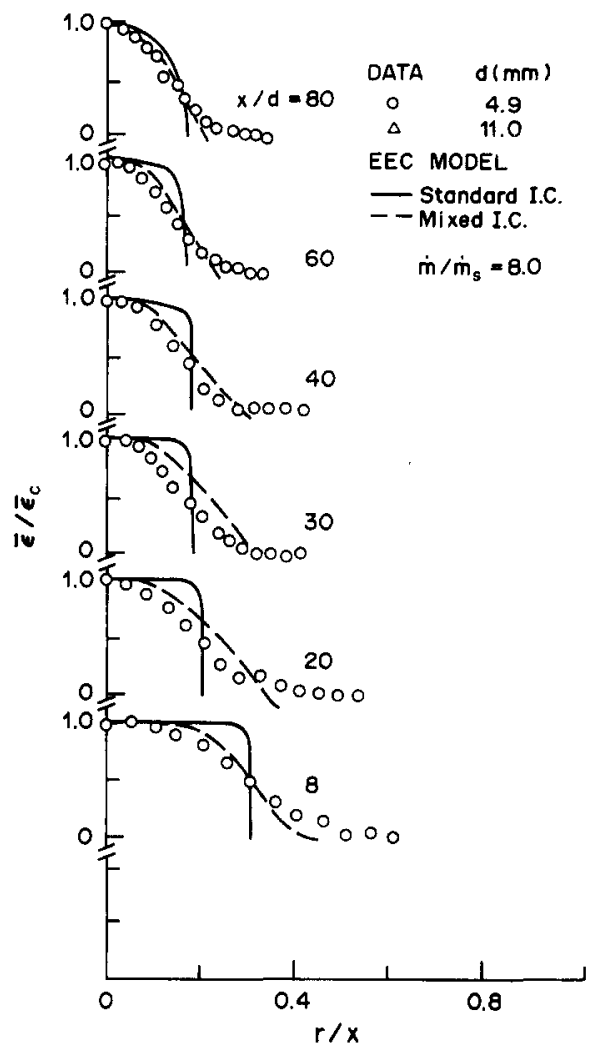

Figure 6. Time-averaged void fractions for a mass flow ratio of 8.0 .

Measured and predicted radial distributions of time-averaged void fractions, $\bar{\epsilon}$ are illustrated in figures 3-6 for $\dot{m} / \dot{m}_{\mathrm{s}}=1.0,2.0,4.0$ and 8.0. Results at $\dot{m} / \dot{m}_{\mathrm{s}}=0.6$ are essentially the same as for $\dot{m} / \dot{m}_{\mathrm{s}}=1.0$ and are reported elsewhere (Loth 1988). The results are plotted as a function of $r / x$, which is the radial similarity variable for fully-developed single-phase turbulent jets, so that the actual width of the flow can be seen. In addition to the present data for 4.9 and $11.0 \mathrm{~mm}$ dia passages, probe measurements from Tross (1974) are also shown for $\dot{m} / \dot{m}_{\mathrm{s}}=1.0$ and 2.0. Similar to the measurements along the axis, the measurements of Tross (1974) are generally lower than present measurements, probably due to probe biases.

The most striking feature of the measurements illustrated in figures 3-6 is the unusual width of the void-fraction profiles. This is expected near the passage exit when results are plotted as a function of $r / x$. Furthermore, at $x / d=4$ and $\dot{m} / \dot{m}_{\mathrm{s}}=2.0$ and 4.0 , there is a bulge in the void-fraction distribution near the edge of the flow which is probably associated with the presence of the external-expansion region at this position (see figure 1). Nevertheless, flow widths remain unusually large even at $x / d=80$, where the flow might be expected to approach the properties of fully-developed jets, e.g. the edge of the gas-containing region is $r / x \sim 0.3$ at $x / d=80$, which is nearly twice the width observed for properties in fully-developed single-phase jets (Wygnanski \& Fiedler 1969). The main reason for this behavior is that the void fraction is an unusually sensitive indicator of the mixing level of the flow at low void fractions. For example, when the mixture fraction $f \ll \rho_{\mathrm{a}} / \rho_{\mathrm{w}}$, the density ratio of air and water, the relationship between the void fraction and mixture fraction becomes

$$
\epsilon=\frac{\rho_{\mathrm{w}} f}{\rho_{\mathrm{a}}}
$$

For the present test conditions $\rho_{\mathrm{w}} / \rho_{\mathrm{a}} \sim 850$; therefore, $\epsilon$ is still significant even when $f$ is much smaller than mixing level values that would normally be associated with the edge of a jet.

In view of the sensitivity of void fractions to mixing levels (mixture fraction), the comparison between predictions and measurements in figures 3-6 is encouraging, in the sense that predicted 
characteristic flow widths tend to scale with the measurements as $x / d$ and $\dot{m} / \dot{m}_{\mathrm{s}}$ vary. However, the evolution of the profiles from the distribution prescribed at the jet exit is relatively slow and profiles using standard initial conditions are far too blunt in the radial direction, particularly near the passage exit. Use of the mixed initial conditions yields much better predictions of the shape of the profiles, particularly near the jet exit. This suggests that mixing is enhanced near the jet exit for all conditions, probably as a result of intrinsic unsteadiness. In particular, failure of the LHF approximation generally causes overestimation of flow widths (Faeth 1987), thus, separated flow phenomena do not provide an obvious explanation of the discrepancies between the predictions and measurements seen in figures 3-6. Similarly, the shapes of the void-fraction profiles do not differ for adapted and underexpanded jets; thus, use of the effective-adapted-jet approximation is not an obvious source of the discrepancies either.

Figure 7 is an illustration of measured and predicted characteristic void-fraction diameters, $2 r_{0.5}$ (defined as twice the radius where $\bar{\epsilon} / \bar{\epsilon}_{\mathrm{c}}=0.5$ ) plotted as a function of $x / d$ for various $\dot{m} / \dot{m}_{\mathrm{s}}$. Also shown on the plot is the radius of the gas-containing region, which is roughly comparable to $2 r_{0.5}$, measured by Bell et al. (1972) using a passage plate having various hole diameters for an underexpansion ratio of 3.7. The results of Bell et al. (1972) are in reasonably good agreement with present measurements in view of the somewhat different definitions of characteristic diameters. The unusual width of these flows, when viewed in terms of $\bar{\epsilon}$, is evident from the figure. As before, this reflects the sensitivity of void fractions to mixing levels (note that a void fraction of 0.5 corresponds to mixture fractions of roughly $0.1 \%$ ). The characteristic void-fraction diameters increase everywhere as the underexpansion ratio increases: even at $x / d=80,2 r_{0.5} / x \sim 0.2$ for $\dot{m} / \dot{m}_{\mathrm{s}}=0.6$ and $\sim 0.3$ for $\dot{m} / \dot{m}_{\mathrm{s}}=8.0$. Predictions using the two initial conditions only differ near the jet exit and are in reasonably good agreement with the measurements.

\section{Dynamic pressures}

Other flow properties exhibit more conventional characteristic flow widths than void fractions since they are less sensitive to mixing levels. This can be illustrated using measurements of dynamic pressures from Tross (1974) for conditions very similar to the present investigation. Although these measurements were obtained using probes, reasonable comparisons between the probe measurements and present nonintrusive measurements of void fractions provide some confidence in the results.

Measured and predicted dynamic pressures along the axis are illustrated in figure 8 . The results involve two initial jet diameters, 3.2 and $6.4 \mathrm{~mm}$, and $\dot{m} / \dot{m}_{\mathrm{s}}=1.0,1.5$ and 2.0 . Although void fractions do not decrease appreciably from unity until $x / d \sim 30$ for these conditions (see figure 2), dynamic pressures begin to decrease before $x / d=10$ and then exhibit a logarithmic decay typical of fully-developed single-phase jets. This difference is a result of the large density ratio of the flow, i.e. small volumes of liquid can absorb large amounts of momentum from the gas. Predictions based on the standard initial conditions tend to overestimate the rate of decay of dynamic pressures; in contrast, use of mixed initial conditions yield reasonably good agreement with the measurementssupporting the presence of unusually high mixing levels near the jet exit. An obvious defect of the predictions, however, is that use of the effective-adapted-jet approximation cannot provide information concerning dynamic pressure variations in the external-expansion region, analogous to the static pressures illustrated in figure 1.

Typical radial distributions of dynamic pressures for underexpanded jets considered by Tross (1974) are illustrated in figure 9. Flow widths exhibited by dynamic pressures are far more typical of the properties of single-phase jets than flow widths based on void fractions, with the edge of the flow at $r / x \sim 0.15$ at $x / d=72$ in figure 9. When normalized in the manner of figure 9 , there is little difference between the predictions using standard and mixed initial conditions and both methods are in good agreement with the measurements.

\section{Entrainment}

Predicted and measured entrainment coefficients, defined according to [2], are plotted in figure 10. Experimental results include: the present measurements; the measurements of Bell et al. (1972) and Carreau et al. (1985) for horizontal underexpanded $\mathrm{N}_{2}$ jets in water, using the water overflow 


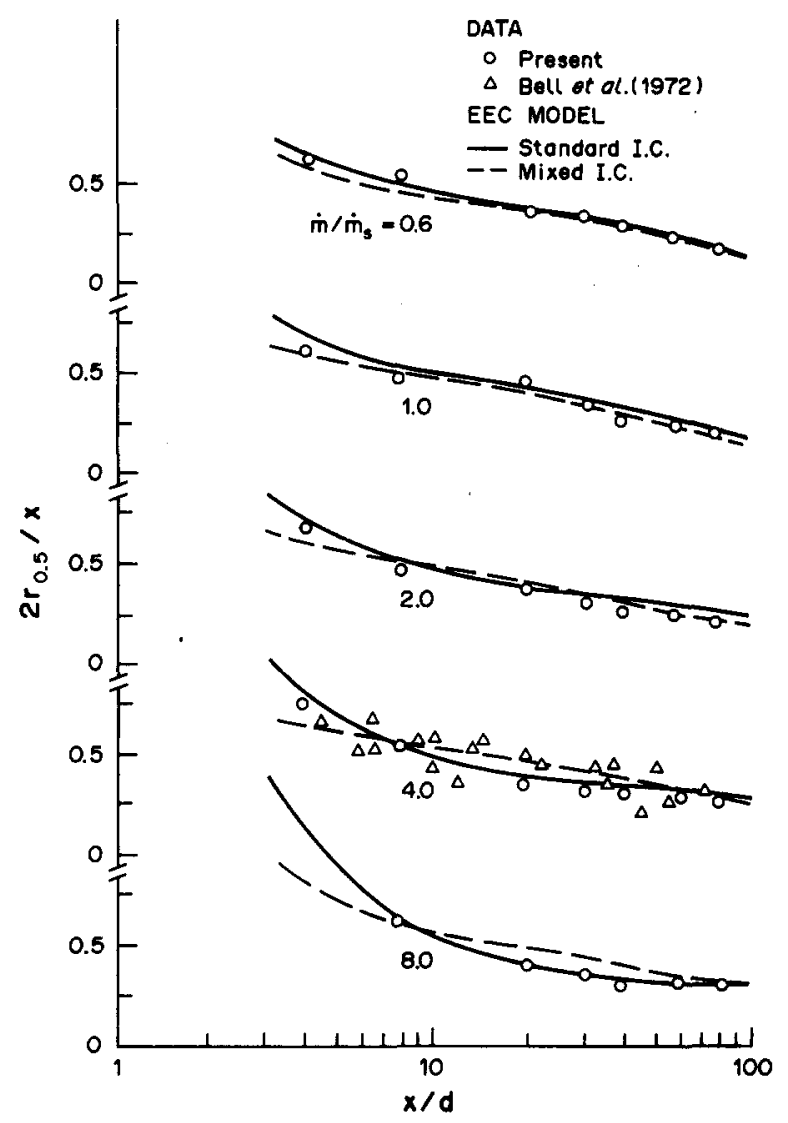

Figure 7. Streamwise variation of the jet width for various mass flow ratios.

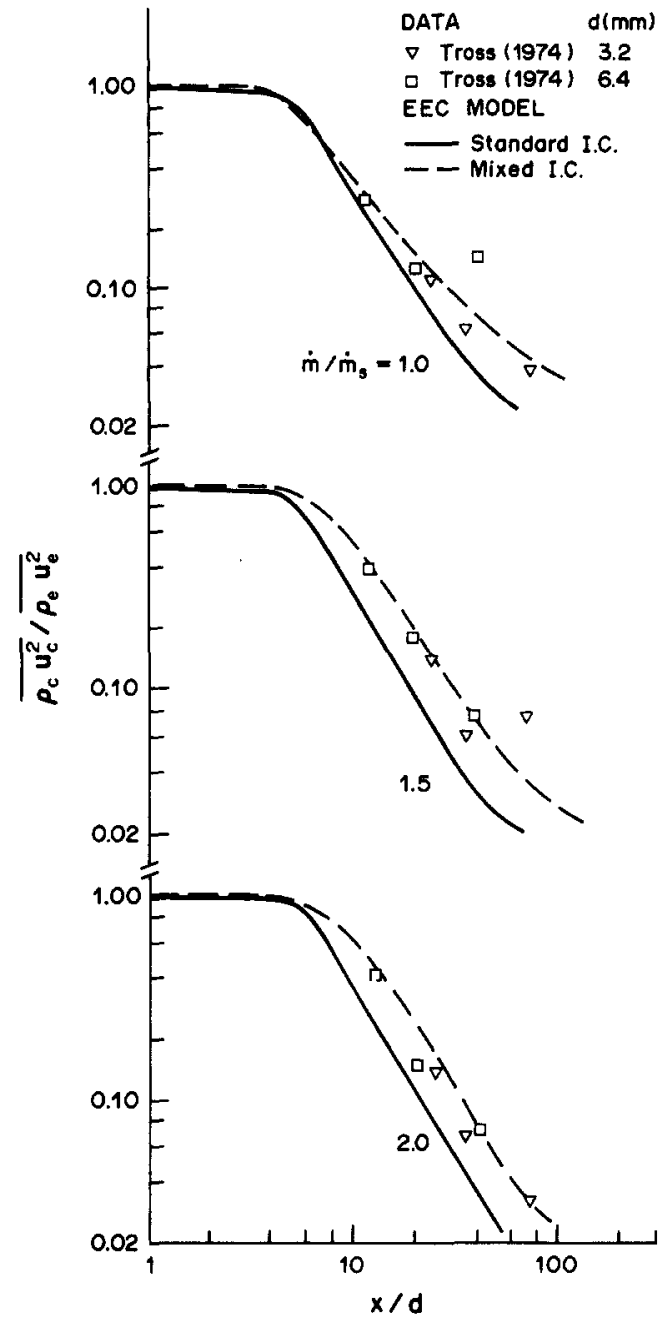

Figure 8. Mean dynamic pressures along the axis for various mass flow ratios: measurements from Tross (1974).

technique; and the empirical correlation of Ricou \& Spalding (1961) for the fully-developed region of single-phase, variable-density gas jets. The present measurements and those of Carreau et al. (1985) are in excellent agreement. The earlier measurements of Bell et al. (1972), however, are more scattered and are generally higher than the rest: the reason for this difference in behavior is not known. Measured entrainment coefficients are relatively constant at small $x / d$ but begin to increase at larger $x / d$, with the increase beginning sooner as $\dot{m} / \dot{m}_{\mathrm{s}}$ is reduced. This is an effect of buoyancy increasing the momentum of the flow, which becomes important nearer to the jet exit as $\dot{m} / \dot{m}$ s decreases, since this reduces the initial streamwise momentum of the flow. When effects of buoyancy are small, the entrainment coefficient remains nearly constant in the fully-developed portion of the flow, similar to the correlation of Ricou \& Spalding (1961). Measured entrainment coefficients also increase everywhere as $\dot{m} / \dot{m}_{s}$ decreases. This is probably due to enhanced mixing caused by increasing unsteadiness at low underexpansion ratios.

The comparison between predictions using standard initial conditions and the measurements, seen in figure 10 , is generally poor, although predictions tend to improve as $\dot{m} / \dot{m}_{\mathrm{s}}$ and $x / d$ increase. Predictions using the mixed initial conditions, however, are in reasonably good agreement with the measurements except for $\dot{m} / \dot{m}_{\mathrm{s}}=\mathbf{0 . 6}$ where predictions substantially underestimate the measurements. This behavior suggests that the discrepancies are largely due to enhanced mixing from effects of unsteadiness very near the jet exit. This is a major effect when the flow at the jet exit is subsonic but still persists to a significant degree for the underexpanded jets. 


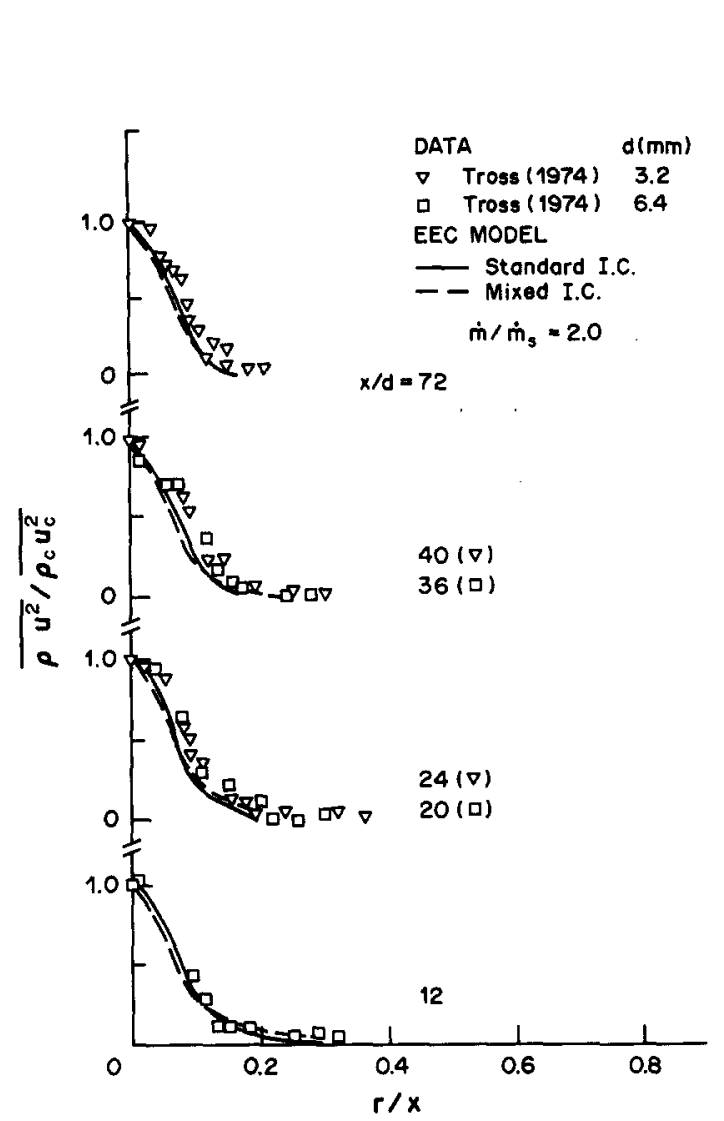

Figure 9. Mean dynamic pressures for a mass flow ratio of 2.0: measurements from Tross (1974).

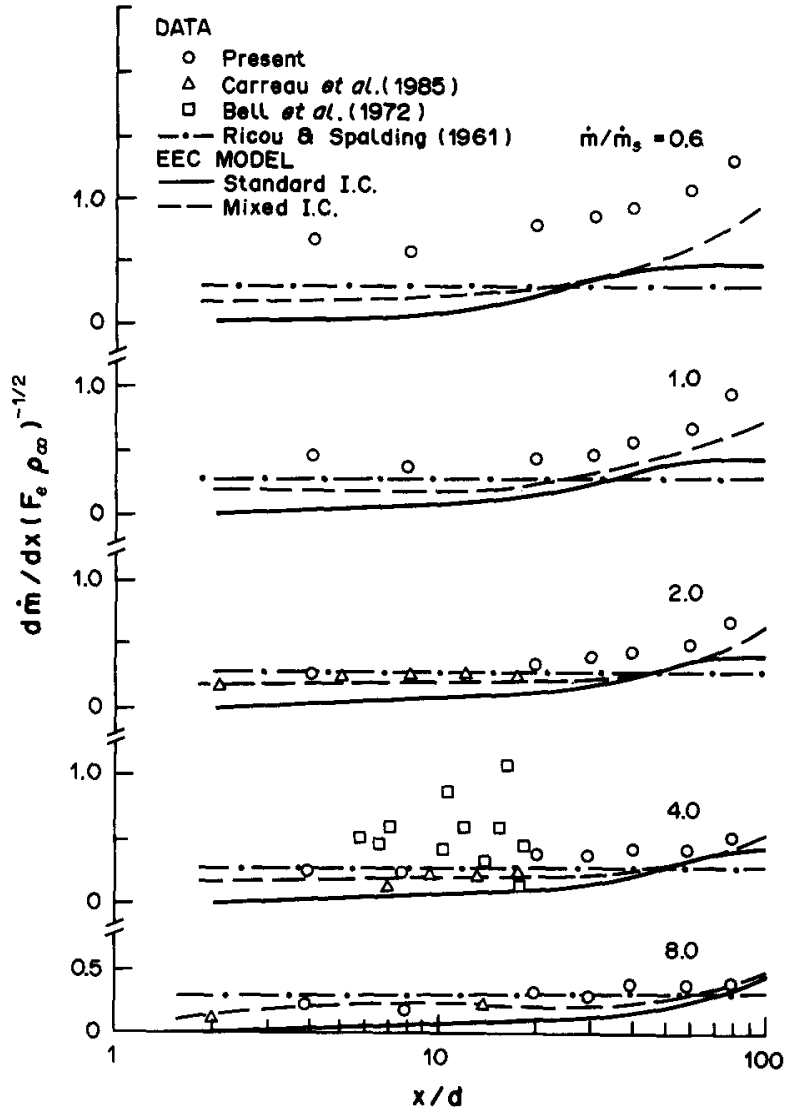

Figure 10. Streamwise variation of the entrainment coefficient for various mass flow ratios.

While the structure and mixing properties of the vertical underexpanded jets considered during the present study and by Tross (1974), and the horizontal underexpanded jets considered by Carreau et al. (1985), are predicted reasonably well using the mixed initial conditions, use of this prescription to allow for near-injector unsteadiness should be approached with caution. In particular, Surin et al. (1983) point out that condensing jets exhibit less unsteadiness than noncondensing jets, while Loth (1988) found that even low levels of liquid coflow can significantly stabilize the flow as well. Clearly, a better understanding of the mechanism of unsteadiness near the jet exit, in the presence of a shock-wave-containing external-expansion region, is required to properly understand and reliably predict the properties of these flows.

\section{CONDITIONS}

The major conclusions of the present study are as follows:

1. A shock-wave-containing external-expansion region is present for underexpanded air jets in water, similar to underexpanded air jets in air, confirming the earlier results of Moiseev (1962) and Surin et al. (1983).

2. Increasing underexpansion ratios, $\dot{m} / \dot{m}_{\mathrm{s}}$, tends to reduce the effects of unsteadiness, as represented by enhanced mixing near the jet exit, however, the penalty for increased stability is slower developement for the multiphase jet, as represented by slower rates of decay of void fractions along the axis.

3. Air jets in water exhibit unusual flow widths based on void fractions due to the strong sensitivity of the void fraction to mixing levels, however, flow widths based on dynamic pressure are more typical of single-phase jets. 
4. Use of the LHF and effective-adapted-jet approximations yielded encouraging predictions of the properties of air jets in water, particularly at large $\dot{m} / \dot{m}_{\mathrm{s}}$ where the effects of unsteadiness were reduced. The prescription of initial conditions for these calculations, however, had a significant influence on predictions of the shape of radial void-fraction distributions and entrainment coefficients. Use of mixed initial conditions, to account for enhanced mixing due to unsteadiness near the jet exit, yielded the best agreement with measurements but additional study is needed to better understand the mechanism of unsteadiness and to establish more rational prescriptions of initial conditions and methods for analyzing flow properties.

Acknowledgements - This research was supported by the Office of Naval Research, Contract No. N00014-850604, with G. D. Roy serving as Scientific Program Advisor.

\section{REFERENCES}

Abdel-Aal, H. K., Stiles, G. B. \& Holland, C. C. 1966 Formation of interfacial area at high rates of gas flow through submerged orifices. AIChE $J l 12,174-180$.

AVERY, J. F. \& FAETH, G. M. 1974 Combustion of a submerged gaseous oxidizer jet in a liquid metal. In Proc. 15th Symp. (Int.) on Combusion, The Combustion Institute, Pittsburgh, Pa, pp. 419-428.

BaKaklevskiI, Y. I. \& CheKhovich, V. Y. 1978 Temperature field of a submerged steam jet. J. Engng Phys. 34, 329-333.

Bell, R. B., Boyce, E. \& Collier, J. G. 1972 The structure of a submerged impinging gas jet. J. Br. nucl. Soc. 11, 183-193.

Bilger, R. W. 1976 Turbulent jet diffusion flames. Prog. Energy Combust. Sci. 1, 87-109.

Birch, A. D., Brown, D. R., Dodson, M. G. \& Swaffield, F. 1984 The structure and concentration decay of high pressure jets of natural gas. Combust. Sci. Technol 36, 249-261.

Birch, A. D., Hughes, D. J. \& Swaffield, F. 1987 Velocity decay of high pressure jets. Combust. Sci. Technol, 52, 161-171.

Carreau, J. L., Loukarfi, L., Gbakoue, L., Hobbes, P. \& Roger, F. 1985 Hydrodynamics of an axisymmetric, submerged non-reactive gas jet-measurement of entrainment-contribution to the wastage modelling. In Proc. 19th IECEC American Nuclear Society, San Francisco, Calif., Paper No. 859176, pp. 1.688-1.695.

Chan, C. K. 1974 Dynamic pressure pulse in stream jet condensation. In Proc. Sth Int. Heat Transfer Conf., ASME, New York, Vol. 3, pp. 226-230.

CHEN, L.-D. \& FAETH, G. M. 1982 Condensation of submerged vapor jets in subcooled liquids. J. Heat Transfer 104, 774-780.

CHEN, L.-D. \& FAETH, G. M. 1983 Structure of turbulent reacting gas jets submerged in liquid metals. Combust. Sci. Technol. 31, 277-296.

Chuech, S. G., LaI, M.-C. \& FAETH, G. M. 1989 Structure of turbulent sonic underexpanded free jets. AIAA Jl 27, 549-559.

CHUN, J. H. \& SonIN, A. A. 1984 Small-scale simulation of vapor discharges into subcooled liquid pools. Presented at the National Heat Transfer Conf., Niagara Falls, N.Y.

Cumo, M., Farello, G. E. \& Ferragi, G. 1987 Direct heat transfer in pressure-suppression systems. Proc. 6th Int. Heat Transfer Conf., ASME, New York, Vol. 5, pp. 101-106.

EGGERS, J. M. 1966 Velocity profiles and eddy viscosity distributions downstream of a Mach 2.2 nozzle exhausting into quiescent air. Report NASA TN D-3601.

FaEth, G. M. 1987 Mixing transport and combustion in sprays. Prog. Energy Combust. Sci. 13, 293-345.

HiNZE, J. O. 1975 Turbulence, 2nd edn, pp. 286-300, 715-742. McGraw-Hill, New York.

JENG, S.-M. \& FAETH, G. M. 1984 Species concentrations and turbulence properties buoyant methane diffusion flames. J. Heat Transfer 106, 721-727.

KERNEY, P. J., FAETH, G. M. \& OlsON, D. R. 1972 Penetration characteristics of a submerged steam jet. $A I C h E ~ J l ~ 18,548-553$. 
Kudo, A., Egusa, T. \& Toda, S. 1974 Basic study on vapor suppression. In Proc. Sth Int. Heat Transfer Conf., ASME, New York, Vol. 3, pp. 221-225.

LAMBieR, G. R. \& ChOw, L. C. 1984 Pressure pulses during vertical and horizontal discharges of stream into subcooled water. Presented at the National Heat Transfer Conf., Niagara Falls, N.Y.

LeE, L., BANKoff, S. G., YUEN, M. C. \& TANKIN, R. S. 1979 Local condensation rate in horizontal co-current steam-water flow. Presented at the 18th National Heat Transfer Conf., San Diego, Calif.

Limbaugh, C. C. \& KNeile, K. R. 1984 Uncertainties propagation for combustion diagnostics using infrared band models. J. quant. Spectrosc. Radiat. Transfer 31, 161-171.

LockwOod, F. C. \& NAGUIB, A. S. 1975 The prediction of the fluctuations in the properties of free, round jet, turbulent diffusion flames. Combust. Flame 24, 109-124.

LoTH, E. 1988 Study of undexpanded turbulent air jets in water. Ph.D. Thesis, The Univ. of Michigan, Ann Arbor.

Mahalingen, R., Limaye, R. S. \& BRINK, J. A. JR 1976 Velocity measurements in two-phase bubble-flow regime with laser-Doppler anemometry. AIChE $J l$ 22, 1152-1155.

MoISEEv, M. G. 1962 Discharge of a gas into a liquid through a Laval nozzle. Inzh.-fiz. Zh. 5(91), $81-84$.

OHBA, K. 1979 Relationship between radiation transmissivity and void fraction in twophase/dispersed flow. Tech. Rep. Osaka Univ. 29, 245-254.

Ohba, K., Kishimoto, I. \& Ogasawara, M. 1977 Simultaneous measurements of local liquid velocity and void fraction in bubbly flows using a gas laser-I. principles and measuring procedure. Tech. Rep. Osaka Univ. 26, 547-556.

Ricou, F. P. \& SPalding, D. B. 1961 Measurements of entrainment by axisymmetrical turbulent jets. J. Fluid Mech. 11, 21-32.

RufF, G. A., SaGar, A. D. \& FAETh, G. M. 1989 Structure and mixing properties of pressureatomized sprays. $A I A A \mathrm{Jl}$. In press.

Santoro, R. J., Semerjian, H. R., Emmerman, P. J. \& Goulard, R. 1981 Optical tomography for flow field diagnostics. Int. J. Heat Mass Transfer 24, 1139-1150.

Schlichting, H. 1979 Boundary Layer Theory, p. 599. McGraw-Hill, New York.

Shapiro, A. 1954 Compressible Fluid Flow, Vol. 1. Ronald Press, New York.

Sherman, P. M., Glass, D. R. \& Duleep, K. G. 1976 Jet flow field due to screech. Appl. scient. Res. 32, 283-303.

Simpson, M. E. \& Chang, C. K. 1982 Hydrodynamics of a subsonic vapor jet in a subcooled liquid. J. Heat Transfer 104, 271-278.

Soo, S. L. 1967 Fluid Dynamics of Multiphase Systems. Blaisdell, Waltham, Mass.

SPALDING, D. B. 1977 GENMIX: a General Computer Program for Two-dimensional Parbolic Phenomena. Pergamon Press, Oxford.

SUN, T.-Y. \& FAETH, G. M. 1986 Structure of turbulent bubbly jets-I. Methods and centerline properties; II. Phase property profiles. Int. J. Multiphase Flow 12, 99-114; 115-126.

Surin, V. A., Erchenko, V. N. \& Rubin, V. M. 1983 Propagation of a gas jet in a liquid. J. Engng Phys. 45, 1091-1101.

TRoss, S. R. 1974 Characteristics of a turbulent two-phase submerged free jet. M.S. Thesis, Pennsylvania State Univ., University Park.

Weimer, J. C., Faeth, G. M. \& Olson, D. R. 1973 Pentration of vapor jets submerged in subcooled liquids. AIChE $J l$ 19, 552-558.

Wygananski, I. \& Fiedler, H. 1969 Some measurements in the self-preserving jet. J. Fluid Mech. 38, 577-612.

Young, R. J., YANG, K. T. \& NovotNY, J. L. 1974 Vapor-liquid interaction in a high velocity vapor jet condensing in a coaxial water flow. In Proc. 5th Int. Heat Transfer Conf., ASME, New York, Vol. 3, pp. 226-230. 